\title{
Megacystis-microcolon-intestinal hypoperistalsis syndrome
}

INSERM

\section{Source}

INSERM. (1999). Orphanet: an online rare disease and orphan drug data base.

Megacystis-microcolon-intestinal hypoperistalsis syndrome. ORPHA:2241

Megacystis microcolon intestinal hypoperistalsis syndrome (MMIHS) is a rare congenital disease characterized by massive abdominal distension caused by a largely dilated nonobstructed urinary bladder (megacystis), microcolon and decreased or absent intestinal peristalsis. 